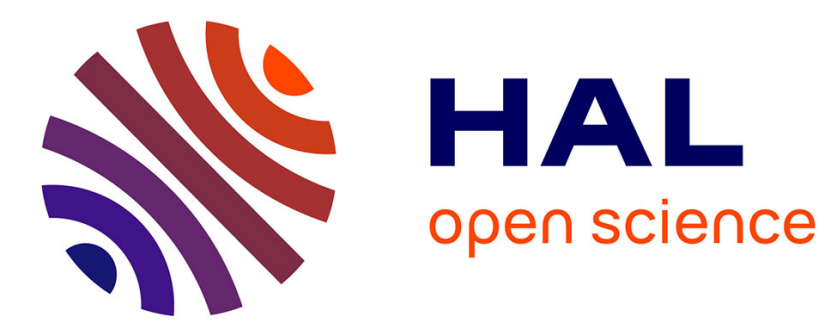

\title{
Ce que le blog apporte à la recherche
}

\author{
Antoine Blanchard
}

\section{To cite this version:}

Antoine Blanchard. Ce que le blog apporte à la recherche. Marin Dacos. Read/Write Book, OpenEdition Press, 2010, Read/Write Book, 9782953641905. hal-01249318

\section{HAL Id: hal-01249318 https://hal.science/hal-01249318}

Submitted on 5 Jan 2016

HAL is a multi-disciplinary open access archive for the deposit and dissemination of scientific research documents, whether they are published or not. The documents may come from teaching and research institutions in France or abroad, or from public or private research centers.
L'archive ouverte pluridisciplinaire HAL, est destinée au dépôt et à la diffusion de documents scientifiques de niveau recherche, publiés ou non, émanant des établissements d'enseignement et de recherche français ou étrangers, des laboratoires publics ou privés.

\section{다)(1) $(5$}

Distributed under a Creative Commons Attribution - NonCommerciall 4.0 International 


\title{
Ce que le blog apporte à la recherche
}

\author{
Antoine Blanchard
}

En 2006, la chercheuse de l'université de Bergen (Norvège) Jill Walker témoignait de sa (déjà) longue expérience du blog académique, en réfutant l'idée répandue selon laquelle le blog est un moyen de documenter la recherche ${ }^{1}$. Même si son blog lui a valu un prix de la Fondation Metzerk au titre de la "dissémination de la science", il lui sert plutôt à mener sa recherche. Elle écrit ainsi : "research happens in blogs, and in the conversations between blogs. Blogs aren't about documentation, they're about doing, thinking and discussing. And they're about catching fleeting thoughts and making them explicit."

Pourtant, le physicien et blogueur Chad Orzel se demandait en octobre 2008 si l'explosion des blogs a vraiment contribué à produire plus de science ${ }^{3}$. Dans son billet et les commentaires qu'il a suscité, la discussion tournait essentiellement autour de la recherche en collaboration, facilitée par le réseautage et l'interactivité du blog. Je vais tenter de montrer qu'à mon sens la contribution du blog à la recherche va plus loin que cela, en m'appuyant sur les travaux de sociologues des sciences qui ont analysé finement ce qui constitue l'activité de recherche. Après avoir observé qu'elle se compose de cinq pôles, Michel Callon, Philippe Larédo et Philippe Mustar de l'École des mines ont proposé l'image d'une rose des vents pour la décrire ${ }^{4}$. Nous allons en suivre les branches une par une, en nourrissant notre réflexion d'exemples essentiellement francophones capables de démontrer la versatilité et l'intérêt du blog en milieu scientifique, en particulier dans les sciences dures et de la nature.

\footnotetext{
${ }^{1}$ Jill Walker, «Not documenting, doing », jill/txt, 06/03/06, http://jilltxt.net/?p=184, consulté le 25 juillet 2009.

${ }^{2}$ Jill Walker, «I won a prize for research blogging!!!! », jill/txt, 06/03/06, http://jilltxt.net/?p=1628, consulté le 25 juillet 2009.

${ }^{3}$ Chad Orzel, «Why Doesn’t Blogging Generate More Science? », [Pourquoi le blog ne génère-t-il pas plus de Uncertain principles, $30 / 10 / 08$, http://scienceblogs.com/principles/2008/10/why_doesnt_blogging_generate_m.php, consulté le 10 juillet 2009. ${ }^{4}$ Michel Callon, Phillipe Larédo, et Philippe Mustar (dir.), La Gestion stratégique de la recherche et de la technologie, 1995, Paris, Economica.
} 


\section{La rose des vents de la recherche}

\section{Connaissances \\ certifiées, instruments}

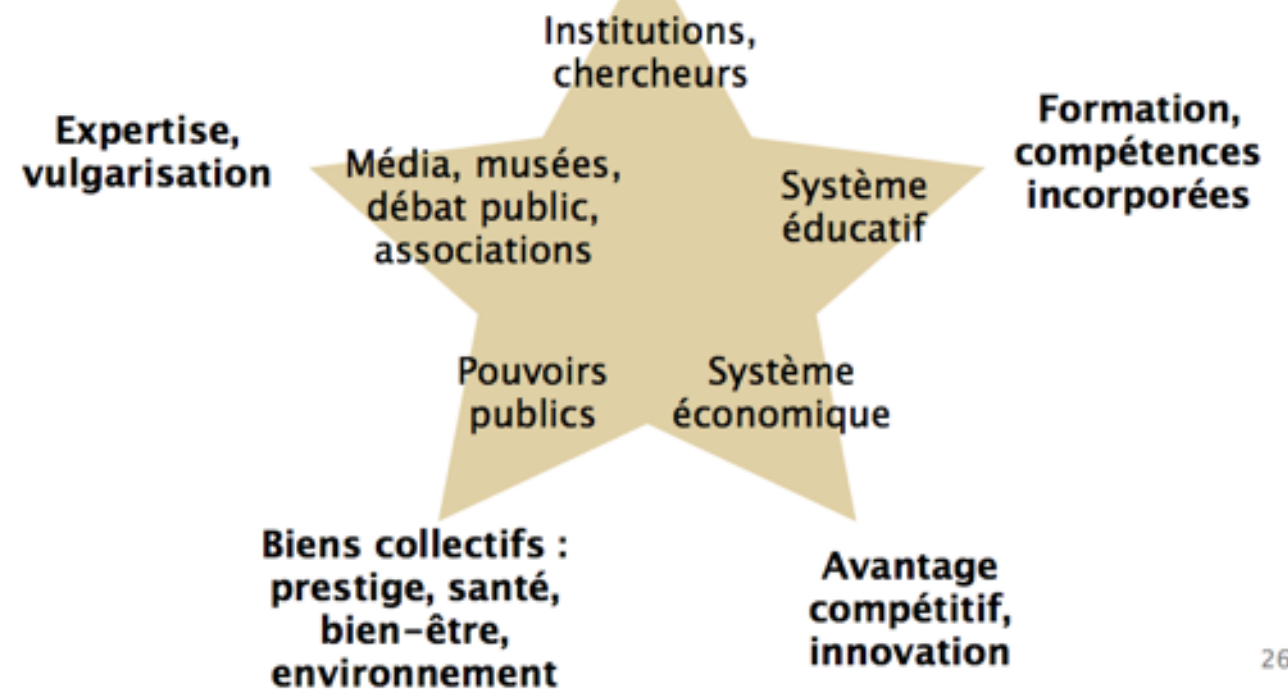

La première de ces branches concerne la production de connaissances certifiées, lesquelles viennent parfois se matérialiser dans de nouveaux instruments destinés à la recherche. Cette production passe évidemment par les institutions de recherche et par la communauté des chercheurs, qu'on appelle les « pairs ». Mais qu'est-ce que le blog apporte à cette activité ?

Partons d'un article scientifique tout ce qu'il y a de plus banal, fruit du processus de production de connaissances certifiées, soumis au jugement des pairs. Cet article ${ }^{5}$ publié en novembre 2005 dans la fameuse revue Plant Cell propose une explication alternative à la nouvelle forme d'hérédité (dite non-mendélienne, car ne respectant pas les règles établies par Gregor Mendel en 1866) observée quelques mois auparavant chez la plante modèle Arabidopsis thaliana ${ }^{6}$.

\footnotetext{
${ }^{5}$ Luca Comai, et Reed A. Cartwright, «A toxic mutator and selection alternative to the non-Mendelian, RNA cache hypothesis for hothead reversion». Plant Cell 17, 2856-2858, 2005, [en ligne], http://www.plantcell.org/cgi/content/full/17/11/2856, consulté le 10 juillet 2009.

${ }^{6}$ Susan J. Lolle, Jennifer L. Victor, Jessica M. Young, et Robert E. Pruitt, « Genome-wide non-mendelian inheritance of extra-genomic information in Arabidopsis ». Nature 434, 505-509, 2005, [en ligne], http://www.nature.com/nature/journal/v434/n7032/full/nature03380.html, consulté le 10 juillet 2009.
} 
Mais on pouvait prendre connaissance de ce morceau de recherche plus de six mois auparavant, en mars 2005, sur le blog de Reed Cartwright ${ }^{7}$. Comme de nombreux blogueurs, ce doctorant avait pris connaissance du résultat étonnant et de tout le bruit qui fut fait autour, puis avait réagit sur son blog en proposant sa vision des choses et en critiquant la revue Nature pour avoir survendu ce résultat. Et l'histoire aurait pu s'arrêter là : ce blogueur a fait connaitre au monde entier son point de vue et passe à autre chose.

Sauf que, et c'est Reed Cartwright lui-même qui le raconte dans un billet ${ }^{8}$, le chercheur Luca Comai a eu exactement la même idée et l'a soumise à la revue Plant Cell. Il fait une recherche Google par acquit de conscience et s'aperçoit qu'il a été scoopé par un blogueur ! Plusieurs options s'offrent à lui et il choisit la plus généreuse et honnête, en offrant à Reed Cartwright de devenir co-auteur de l'article. Celui-ci accepte, tout heureux de ce concours de circonstances qui lui offre sur un plateau son premier article scientifique ! Ce que montre cette histoire, c'est comment les frontières se brouillent entre billet de blog et article académique, qui peuvent proposer le même contenu et sont traités indifféremment par les moteurs de recherche Internet, mais aussi comment le blog accélère la recherche en s'affranchissant du délai nécessaire pour écrire, soumettre et publier un article académique. L'histoire suivante, empruntée au blog À la source ${ }^{9}$, le montre encore mieux.

Tout démarre quand Xian-Jin Li publie sur le site de pré-publications arXiv une preuve de la mythique hypothèse de Riemann ${ }^{10}$, qui résiste depuis 150 ans aux mathématiciens du monde entier et dont la résolution est assortie d'un prix d'un million de dollars offert par le Clay Mathematical Institute. Nous sommes alors le 1er juillet 2008.

Le lendemain, un lecteur du blog du mathématicien et lauréat de la médaille Fields Terence Tao laisse un commentaire ${ }^{11}$ pour signaler cette pré-publication, en ajoutant que ce n'est pas un canular vu la réputation de l'auteur. Un jour plus tard, le 3 juillet, Terence Tao répond en expliquant qu'il y a une erreur dans l'équation (6.9) de la page 20 et que la démonstration ne tient pas.

Entre temps, un autre commentaire ${ }^{12}$ est déposé chez Alain Connes, fameux mathématicien et autre lauréat de la médaille Fields, pour attirer l'attention des lecteurs vers la pré-publication de Xian-Jin Lin. Alain Connes répond dans la foulée et souligne une incohérence à la page 31 . Nous sommes alors le 3 juillet.

Le 6 juillet, Xian-Jin Lin retire la quatrième version de son article ${ }^{13}$, amendé entre temps à cause d'une erreur à la page 29. En l'espace de quelques jours et grâce à la combinaison d'un entrepôt de pré-publications et des blogs, ce travail potentiellement révolutionnaire aura donc été porté à la connaissance de la communauté scientifique, analysé, modifié et définitivement traité. Surtout, la blogosphère ne s'est pas limité à l'avis «éclairé » de quelques mathématiciens reconnus et a arbitré, commenté, pris position etc. La recherche, elle, peut poursuivre sa route, l'intégralité de ces échanges restant disponible grâce aux rétroliens fournis par arXiv qui permettent de retrouver les blogs qui ont discuté la pré-publication.

\footnotetext{
${ }^{7}$ Reed A. Cartwright, «Existence of RNA Genome or Fertility Selection?», De Rerum Natura on the nature of thing, 23/03/05, http://www.dererumnatura.us/archives/2005/03/existance_of_rn.html, consulté le 10 juillet 2009 .

${ }^{8}$ Reed A. Cartwright, «Blog About Hothead and Get an Easy Paper? », De Rerum Natura on the nature of thing, 03/11/2005, http://dererumnatura.us/archives/2005/11/blog_about hoth.html, consulté le 10 juillet 2009.

9 David Larousserie, «Butinage (10)», $\grave{A}$ la source, 17/07/2008, http://alasource.aliceblogs.fr/blog/_archives/2008/7/17/3797041.html, consulté le 10 juillet 2009.

${ }^{10}$ Xian-Jin $\mathrm{Li}$, «A proof of the Riemann hypothesis », arXiv.org, 01/08/09, http://arxiv.org/abs/0807.0090v1, consulté le 10 juillet 2009.

${ }^{11}$ http://terrytao.wordpress.com/2008/02/07/structure-and-randomness-in-the-prime-numbers/\#comment-30708, consulté le 10 juillet 2009.

12

http://noncommutativegeometry.blogspot.com/2008/06/fun-daytwo.html?showComment=1215053400000\#c7113256183353024311, consulté le 10 juillet 2009.

${ }^{13}$ http://arxiv.org/abs/0807.0090, consulté le 10 juillet 2009.
} 
Dans un autre registre, le laboratoire de Jean-Claude Bradley à l'université Drexel (ÉtatsUnis) tient un blog ${ }^{14}$ sur lequel il publie le résultat de ses expériences de chimie de synthèse, qu'elles aient fonctionné ou non. Ce travail est mis intégralement à disposition de la communauté, sous une licence libre qui autorise qu'il soit repris et réutilisé, le but étant d'empêcher le dépôt de brevets et de mutualiser les efforts pour obtenir une substance antipaludique. Ici, le blog s'insère de fait dans un ensemble plus vaste (wiki ${ }^{15}$, expériences filmées ${ }^{16} \ldots$ ) qui correspond à cette « science 2.0 » à la fois ouverte, en ligne et participative.

L'exemple suivant est directement tiré de mon blog. En mai 2008, je publiais le fruit d'une réflexion en cours ${ }^{17}$, dont j'annonçais que j'espérais la conduire jusqu'à la publication d'un article et que j'incitais à commenter et discuter largement. La réflexion en question portait sur les façons de montrer la science «en train de se faire », avec sa part humaine et chaude, plutôt que la science « déjà faite » comme un savoir livresque. J'approchais cette thématique avec un regard très sociologique et les discussions en commentaires furent très riches.

En particulier, le commentaire de Matteo Merzagora ${ }^{18}$ souligna que mon point de vue emprunté à la sociologie des sciences me faisait occulter toute la littérature en muséologie. Admettant ne pas être du tout familier avec cette recherche, je me suis plongé aussitôt dans les références indiquées et $\mathrm{j}$ 'ai pu notablement enrichir mon travail pour le soumettre à une revue à comité de lecture. Je dois donc à mon blog d'avoir servi de brouillon ou de carnet de recherche pour un travail en cours et d'avoir pu croiser les regards disciplinaires. Sans cela, je n'aurais pas pensé par moi-même à me pencher sur la muséologie et à faire un tour à la bibliothèque ou à interroger un spécialiste. Même un rapporteur aurait pu passer à côté de ces références inhabituelles.

Arrivé après les blogs, l'outil Friendfeed ${ }^{19}$ nous fait passer à l'étape du micro-blogging (qui permet de publier des réflexions condensées, à la façon de Twitter ou du statut Facebook) mais surtout du lifestreaming (qui regroupe l'ensemble des flux composant son moi numérique : signets, photos, blogs, commentaires...). Un peu comme sur Facebook, on choisit de s'abonner à certains profils en particulier et réciproquement, on peut savoir qui nous « suit », renforçant ainsi le sentiment de communauté. Friendfeed est notamment peuplé de biologistes 2.0, une communauté très vivante qui interagit d'une façon probablement inédite (pour un aperçu, voir le groupe «The Life Scientists ${ }^{20}$ ). Sous le titre «Request for assistance », le biochimiste Cameron Neylon a par exemple utilisé le crowdsourcing pour trouver un collaborateur ${ }^{21}$ : il aurait besoin de modéliser le canal potassium MthK mais ne possède pas l'expertise en interne, et propose en échange de toute aide une co-signature sur l'article qui en résulterait. Un spécialiste de modélisation a répondu et le travail a pu être mené à bout.

\footnotetext{
${ }^{14} \mathrm{http}: / /$ usefulchem.blogspot.com/

${ }_{15}^{15} \mathrm{http:// \text {usefulchem.wikispaces.com/ }}$

${ }_{16}^{16}$ http://www.jove.com/index/details.stp?ID=942

${ }_{17}$ Antoine Blanchard, «Comment montrer la "science en train de se faire" ? », La science, la cité, 31/05/08, http://www.enroweb.com/blogsciences/index.php?2008/05/31/261, consulté le 10 juillet 2009.

$18 \mathrm{http}: / / \mathrm{www} . e n r o w e b . c o m / b l o g s c i e n c e s / i n d e x . p h p ? 2008 / 05 / 31 / 261$-comment-montrer-la-science-en-train-dese-faire\#c11235, consulté le 10 juillet 2009.

${ }^{19} \mathrm{http} / / /$ friendfeed.com/

${ }^{20} \mathrm{http://friendfeed.com/rooms/the-life-scientists}$

${ }^{21} \mathrm{http} / / /$ friendfeed.com/cameronneylon/9875b15c/request-for-assistance
} 
Finalement, le blog permet de faire comme à chaque congrès scientifique, mais à une échelle planétaire et en continu : réseauter. Prendre des nouvelles des uns et des autres, savoir comment avance le travail d'un ancien collègue, sentir les nouveaux sujets à la mode, pour pouvoir choisir son sujet de recherche ou sa prochaine collaboration en toute connaissance de cause. Un des murs de la Royal Institution londonienne qui accueillait le congrès Science Blogging 2008 reproduisait cette citation d'Earl Wilson en grands caractères : " La pause café pourrait bien être le meilleur système de communication que la science se soit trouvée ». Le blog, parce qu'il permet aussi de lancer des idées et de discuter sur un mode semi-informel, pourrait bien devenir la pause café du XXI ${ }^{\mathrm{e}}$ siècle !

De retour vers la rose des vents, nous voyons qu'une dimension importante de la recherche concerne la formation, qui permet d'incorporer dans le corps social les savoirs et compétences développées au laboratoire. Même question : comment le blog peut-il être utile de ce point de vue ?

Prenons à titre d'exemple le blog Cours à l'IUT de La Roche-sur-Yon ${ }^{22}$. Ce «carnet pédagogique », pour reprendre le terme de l'enseignant qui le tient, prolonge le cours en mettant à disposition des étudiants, polycopiés, exercices, liens vers des ressources complémentaires constamment mis à jour, et autorise des échanges autour de certains points qui nécessiteraient discussion. Le tout à la face du monde, afin d'en faire profiter d'autres et d'inviter chacun à enrichir la réflexion pédagogique.

Cet exemple n'a pas été pris au hasard. Derrière cette expérience éducative, on retrouve le chercheur en sciences de l'information Olivier Ertzcheid. Sur son blog Affordance.info ${ }^{23}$, il s'engage activement en faveur d'une pénétration plus importante des blogs dans le milieu académique, et livre quelques réflexions dans ce sens. Ainsi, il rapporte dans un billet ${ }^{24}$ les propos d'Henry Jenkins au MIT, qui dispose de quelques années de recul de plus que nous sur le sujet. Ce que constate Jenkins, c'est que les étudiants (stagiaires, doctorants...) qui rejoignent les laboratoires du MIT ont une vision de plus en plus fine de ses activités : en plus d'en avoir lu les publications scientifiques et de connaître son curriculum, ils savent sur quels sujets de recherche le laboratoire est engagé, comment le travail s'organise... Soit « autant de temps gagné pour l'intégration dans un programme de recherche et l'avancée dudit programme ».

Un troisième volet de l'activité de recherche est lié à l'innovation, qui constitue sa dimension proprement économique. Cela inclut l'entrepreneuriat, le transfert de technologies, la R\&D, la compétition sur un marché... Un monde que l'on penserait à l'opposé du blog, et pourtant ! Une recherche Google avec des mots clés très techniques comme « réduction bruit vibrations » donne, chose étonnante, un blog ${ }^{25}$ dans les premiers résultats.

\footnotetext{
${ }^{22} \mathrm{http}: / /$ oecoursiut.wordpress.com/

${ }^{23} \mathrm{http}: / /$ affordance.typepad.com/

Olivier Ertzcheid, «Les universitaires devraient blogguer», Affordance.info, http://affordance.typepad.com/mon_weblog/2008/04/les-universitai.html, consulté le 11 juillet 2009.

09/04/08, ${ }^{25}$ http://bruit-vibrations.blogspot.com/
} 
Il s'agit en l'occurrence du blog d'un jeune ingénieur d'étude en mécanique, qui offre « références bibliographiques, outils théoriques, approches méthodologiques et solutions techniques pour la réduction du bruit et des vibrations mécaniques ». Le contenu est très professionnel et l'abondance de mots clés («vibration », « résonance », « énergie », «bruit », « automobile », «matériau », « viscosité », «fréquence », « onde», «frottement »...) le rend parfaitement indexé par Google, d'autant plus qu'il est hébergé par la propre plateforme de blogs du moteur de recherche, Blogger. Ainsi, ce jeune ingénieur possède une visibilité notoirement accrue sur Internet, ce qui en fait une autorité de fait et un probable contact pour tout industriel qui s'intéresserait à ce sujet.

Je ne suis pas le premier à souligner cet avantage. Samuel Bouchard, qui s'intéresse à la technologie et à l'entrepreunariat, s'est $\operatorname{aperçu}^{26}$ que son diaporama de thèse sur les robots conduits par câble (cable-driven robots) ressortait deuxième sur Google après une recherche sur ces mots-clés. Là encore, même principe : en mettant son travail sur la plateforme en ligne Slideshare et en lui déléguant le soin d'optimiser l'indexation par les moteurs de recherche, Samuel Bouchard établit son autorité sur ce sujet de recherche et accapare la visibilité vis-àvis d'interlocuteurs éventuels.

Indissociable de la recherche, et pendant de la sphère économique, on trouve dans la quatrième branche de la rose des vents des objectifs qui sont ceux des pouvoirs publics et qui correspondent à ce que les économistes appellent des biens collectifs : puissance, prestige, bien-être (environnement, santé...). La place du blog dans cet écosystème tient essentiellement à celle des citoyens et des groupes concernés.

Prenons l'exemple d'un blog consacré aux myopathies de Bethlem et Ullrich ${ }^{27}$. Mis en place par une association de patients et de familles de patients, il permet d'abord de s'informer sur ces maladies. Ainsi, un billet récent interrogeait Paolo Bernardi, spécialiste du mécanisme moléculaire conduisant à la mort musculaire dans ces myopathies. Grâce aux commentaires, on peut imaginer que le blog permette d'aller plus loin, en faisant interagir médecins, chercheurs et patients et en devenant un véritable outil de circulation de la parole. [NB : En juillet 2009, sept mois après avoir écrit les lignes ci-dessus, Bethlem.info est devenu une plateforme réunissant des patients atteints par la maladie et un site d'information. La forme blog a été abandonnée au profit d'un site communautaire servant ce nouveau projet.]

Le projet d'Institut des épilepsies de l'enfant et de l'adolescent (IDEE), lui, s'inscrit avant tout dans le monde réel et vise à fédérer médecins, chercheurs, entreprises et patients pour faire reculer cette maladie. En amont de cet institut dont le financement est voulu dans un futur proche, un $\operatorname{blog}^{28}$ a été ouvert pour communiquer mais aussi pour permettre à ces participants hétérogènes d'interagir dans un espace neutre et accessible à tous.

Enfin, la dernière dimension de la recherche est celle de l'expertise et de la diffusion dans l'espace public, qui passent par les médias mais aussi les musées, les associations, les instances de débat public etc. C'est l'apport le plus évident du blog, alors que sa contribution réelle n'est pas forcément bien connue.

\footnotetext{
${ }^{26}$ Samuel Bouchard, «Utiliser le Web pour promouvoir ses recherches », Le Lablogatoire, 08/09/08, http://www.lablogatoire.com/2008/09/08/utiliser-le-web-pour-promouvoir-ses-recherches/, consulté le 10 juillet 2009.

${ }^{27} \mathrm{http}: / / \mathrm{www} \cdot$ bethlem.info/

${ }^{28} \underline{\text { http://www.blog-idee.org/ }}$
} 
Les formes traditionnelles de vulgarisation (émissions de télé, musées, Fête de la science...) s'adressent à un public curieux et varié, mais les enquêtes montrent qu'il est très stéréotypé : beaucoup de catégories socio-professionnelles supérieures, des scolaires et des quadragénaires ou plus. À l'inverse, le blog est accessible à tous (internautes) et même une personne récalcitrante et absolument pas au fait des blogs de science peut y atterrir à la suite d'une recherche sur Google. Ainsi, la fameuse blogueuse américaine GrrlScientist rapporte dans un entretien ${ }^{29}$ que deux tiers de ses visiteurs ont une affiliation gouvernementale ou académique (adresses en .edu ou .gov) mais qu'elle reçoit aussi des sénateurs, des éditeurs scientifiques, des juristes, des financiers, des adolescents et des grands-parents, ainsi qu'une classe entière qui lit son blog de façon régulière.

Faisons un point sur la place de la science dans les médias. Selon une étude belge ${ }^{30}$, sa part dans les journaux va de presque $20 \%$ à moins de $5 \%$. C'est très hétérogène et malgré la performance du Vif/L'Express, on peut probablement faire beaucoup mieux.

Quant au contenu de cette couverture médiatique, il est fait pour près de $50 \%$ de médecine, le reste se partageant équitablement entre la science et la technologie. Une personne qui se désintéresse des nouvelles médicales se retrouve donc avec une moitié de contenu en moins ! Ainsi, non seulement il y a de la place pour parler de plus de science, mais il y en a pour parler de la science autrement, en variant le contenu et en explorant des voies de traverse. Le blog, comme outil de publication non mainstream, s'y prête à merveille.

\footnotetext{
${ }^{29}$ Joanne Hinkel, «The Science Buzz: Bloggers Bring Science Ed to the Public », Under the microscope, 07/10/08, http://www.underthemicroscope.com/index.php?option=com_content\&task=view\&id=87\&Itemid=54, consulté le 10 juillet 2009.

${ }^{30}$ Christine Servais (dir.), La place de la science dans la presse écrite belge et francophone. État des lieux et propositions, Bruxelles, Politique scientifique fédérale, 2006, [en ligne] http://www.belspo.be/belspo/home/publ/pub_ostc/Journ/rappULg_fr.pdf, consulté le 10 juillet 2009.
} 
Préférez-vous que les informations scientifiques vous soient présentées par...

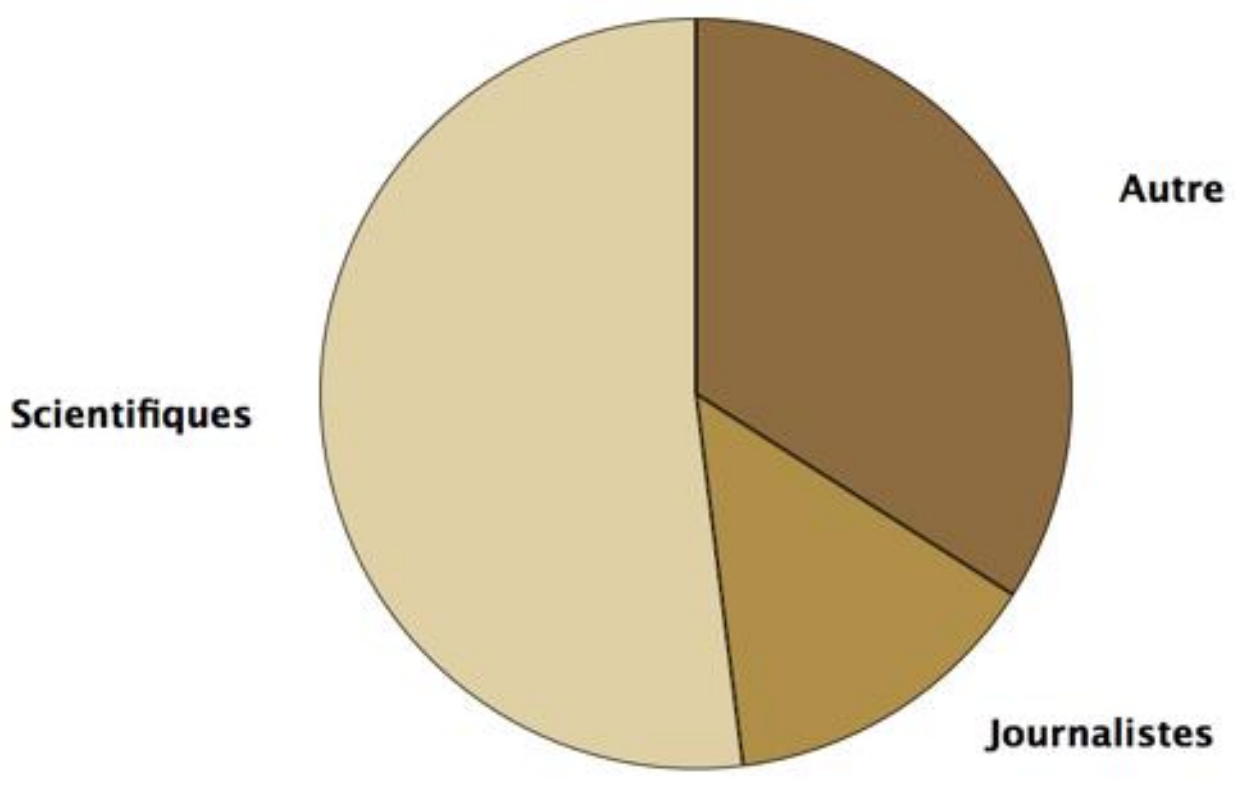

Eurobaromètre Spécial 282, décembre 2007, p. 16

Selon un sondage Eurobaromètre de décembre $2007^{31}, 52 \%$ des sondés préfèrent que les chercheurs eux-mêmes, plutôt que des journalistes, leur présentent les informations scientifiques. Le blog, qui est justement une tribune au service du chercheur, peut remplir cette fonction et répondre à une vraie demande. En tous cas, il y a là une opportunité pour les chercheurs qu'il serait dommage de ne pas saisir !

Une autre caractéristique du discours scientifique sur les blogs est qu'il est ouvert à la contestation et la discussion, bien loin du modèle traditionnel descendant (top-down) où la parole de l'expert est à prendre telle quelle et où elle n'engage aucune discussion. Cela peut prendre de nombreuses formes, de la controverse au simple signalement en passant par l'approbation, la demande de précisions, l'interrogation, la réfutation, le complément... Cette conversation reflète la diversité des sujets et des points de vue qui sont échangés, avec en ligne de mire la curiosité et le plaisir.

\footnotetext{
${ }^{31}$ Eurobaromètre spécial 282. La recherche scientifique dans les médias, Bruxelles, Commission européenne, 2007, [en ligne] http://ec.europa.eu/public_opinion/archives/ebs/ebs_282 fr.pdf, consulté le 10 juillet 2009.
} 
Et puis le blog a des effets de bord, entraînant souvent le blogueur dans un cercle vertueux : plus il blogue plus il devient visible et sollicité mais aussi plus sa plume s'affûte et plus il est pertinent et lu. Baptiste Coulmont, maître de conférence en sociologie à l'université Paris 8, raconte ainsi dans un article de l'AMUE ${ }^{32}$ comment il est passé sur France 3 et a été invité à un colloque sur Singapour en remplaçant au pied levé un intervenant, parce que son blog $^{33}$ lui donne une bonne visibilité sur Internet. Mais la difficulté consiste souvent à obtenir le coup de pouce initial qui permet ensuite de se démarquer, plutôt que de rester dans sa niche en touchant un nombre restreint de visiteurs.

Le monde de la recherche a trouvé une solution à cette difficulté : il suffit de se présenter aux grandes messes qui attirent l'ensemble des chercheurs de sa communauté, pour y présenter ses résultats sous forme de poster à défaut de pouvoir intervenir à l'oral. À chaque congrès, ceux qui présentent un poster et leur public sont concentrés au même endroit au même moment, ce qui est idéal pour toucher le plus de monde possible. Et puis la fin du congrès arrive, les chercheurs rangent leurs posters dans leurs tubes et ils les affichent dans le couloir du laboratoire à leur retour. Là, ils seront vus par une poignée de personnes chaque semaine, des visiteurs du laboratoire déjà au courant de ses activités plutôt qu'un public large et non intéressé a priori.

De la même façon, un chercheur ou un laboratoire qui veut ouvrir un blog peut le faire sur le site web de son département ou institution mais il ne touchera sans doute qu'un public déjà informé et ne se fera pas connaître au-delà. À l'inverse, s'il s'installe directement là où sont les lecteurs, c'est-à-dire une plateforme spécialisée ou un portail qui rassemble un grand nombre de visiteurs, il a toutes les chances de se faire remarquer, comme son poster à un congrès.

Dans le monde francophone, il y a encore peu de solutions pour promouvoir les initiatives de blogs scientifiques. Le C@fé des sciences est un agrégateur de blogs de sciences dures et de la nature, qui compte 25 membres en juillet $2009^{34}$. Cette communauté peut servir de "plateforme de lancement" pour tout blog mais s'ouvre aux nouveaux membres en fonction de critères assez stricts, destinés à maintenir l'exigence de qualité et la ligne éditoriale orientée vers le public le plus large possible. Encore plus fermé est le portail de l'Agence sciencepresse, intitulé Science ! On blogue, proposant dix blogs tenus par des chercheurs québecois démarchés directement par les administrateurs ${ }^{35}$. Quelques universités comme Paris 5 ou Rennes 1 mettent à disposition de leurs personnels et étudiants un hébergement de blogs. Enfin, du côté des sciences humaines et sociales, il faut signaler la plateforme de carnets de recherche Hypothèses pilotée par le Centre pour l'édition électronique ouverte (Cléo / Revues.org), qui offre un hébergement clés en mains à défaut de construire pour l'instant une vraie communauté ${ }^{36}$. Ce qu'il manque en particulier au paysage francophone, ce sont des outils comme Postgenomic ${ }^{37}$ ou Research Blogging ${ }^{38}$ qui permettent d'organiser et de rendre encore plus intelligent ce contenu produit en quantité de plus en plus grande par les chercheurs (et les amateurs de science). Espérons que nous n'avons plus très longtemps à attendre!

\footnotetext{
${ }^{32}$ Agence de mutualisation des universités et établissements, « Blogs et enseignement supérieur », 17/01/06, [en ligne] http://www.amue.fr/systeme-dinformation/metier/articles/article/blogs-et-enseignement-superieur/, consulté le 10 juillet 2009.

${ }^{33} \mathrm{http}: / /$ coulmont.com/blog/

34 http://www.cafe-sciences.org/

35 http://blogue.sciencepresse.qc.ca/

36 http://hypotheses.org/

${ }^{37} \mathrm{http}: / / \mathrm{www}$.postgenomic.com/

38 http://www.researchblogging.org/
} 